\title{
AMOSTRAGEM EM DOIS ESTÁGIOS COM UNIDADES PRIMÁRIAS DE TAMANHOS DIFERENTES
}

\section{SUBSAMPLING TO TWO PROBATION WITH PRIMARY UNITS OF UNEQUAL SIZES}

\author{
Sylvio Péllico Netto ${ }^{1}$
}

\begin{abstract}
RESUMO
Este trabalho enfoca a metodologia de alocação da amostragem dentro de um estrato, em uma população florestal plantada. O critério de alocação considerou os talhões como parte intrínseca do processo estatístico aplicado à amostragem. A eficiência da metodologia foi buscada considerando-se a variação do tamanho dos talhões e, conseqüentemente, efetuando-se a alocação da amostragem proporcional aos seus respectivos tamanhos. O resultado da alocação mostrou-se promissor e apropriado para aplicação aos inventários florestais contínuos.
\end{abstract}

Palavras-chave: Amostragem, Inventário Contínuo.

\section{SUMMARY}

This paper deals with the methodology of sampling allocation in forest stratification. The criteria used for the allocation includes the participation of stands inside a stratum as part of the sampling procedure, known in the literature as "Subsampling With Units of Unequal Sizes". The efficiency of this applied methodology was considered due to the stand's size variation and, consequently, doing to the subsampling allocation with selection proportional to their sizes. The results of this procedure showed very promising and appropriated to be used in continuos forest inventories.

Key words: Sampling, Continuos Inventory.

\section{INTRODUÇÃO}

O processo de amostragem a ser aplicado dentro de cada estrato de uma população florestal, constitui um dos importantes componentes do inventário florestal contínuo.

1. Professor Senior da UFPR, Professor Adjunto da PUC-PR e Pesquisador 1-C do CNPq. 
As áreas reflorestadas geralmente são planejadas administrativamente e são formadas de unidades mínimas, denominadas talhões, geralmente de tamanhos variáveis e, portanto, serão denominadas de unidades primárias da amostragem. Estas unidades permitem ao manejador utilizálas como unidades de planejamento da produção florestal, dentro das quais são efetuadas um segundo estágio da amostragem, geralmente denominado de subamostragem.

Do ponto de vista estatístico, fica caracterizado nesta estrutura, fundamentalmente, um processo de amostragem em dois estágios, com unidades primárias de tamanhos desiguais.

$\mathrm{Na}$ maioria das empresas, as discussões sobre este tema não têm sido efetuadas por dois motivos básicos: o primeiro é que, normalmente, se tem uma baixa intensidade de amostragem dentro do estrato, de tal forma a não se incluir os talhões como partes integrantes dos estimadores estatísticos por estrato; o segundo refere-se a utilização apenas de uma estrutura aleatória dentro dos estratos, onde as unidades amostrais se distribuem internamente dentro dos talhões, mas sem se caracterizar o processo como dois estágios para a alocação da amostragem.

As implicações decorrentes da caracterização dos talhões como fase integrante do processo de amostragem a ser estruturado dentro dos estratos, serão tratadas no contexto deste trabalho.

\section{CRITÉRIO APLICATIVO DA AMOSTRAGEM}

A seleção da amostragem nas condições já expostas, poderá ser efetuada segundo critérios, cuja efetividade dependerá das condições distributivas da variável em análise. Existem, basicamente, duas alternativas para esta seleção assim caracterizadas:

a) Seleção da amostragem com igual probabilidades;

b) Seleção da amostragem com probabilidades desiguais.

SUKHATME et. al. (1984), COCHRAN (1963) e FAO (1973) discutem amplamente estas alternativas para a seleção da amostragem através deste processo e o caso da aplicação em foco, se inclui, preferencialmente, no de seleção com probabilidades desiguais, dado sua maior eficiência se fundamentar na ocorrência de correlação entre a variável $\mathrm{X}_{\mathrm{i}}$ - volume de madeira por unidade de área - e o tamanho da unidade primária $\mathrm{M}_{\mathrm{i}}$. É fácil admitir, que quanto maior for o tamanho dos talhões, tanto maior será a chance de variarem os sítios e, conseqüentemente, $\mathrm{X}_{\mathrm{i}}$. Nessas circunstâncias, afirma COCHRAN (1963), existe uma relação de proporcionalidade entre $V_{x_{i}}$ e $N_{i}^{-g}$ e, quando $\mathrm{g}<1$, a seleção com probabilidade proporcional a um tamanho leva a maior precisão na obtenção dos estimadores estatísticos. O mesmo autor afirma também que a condição de $\mathrm{g}<1$ ocorre na maioria das vezes.

Baseando-se numa prévia aceitação destas condições, sugere-se que a amostragem seja efetuada através do processo denominado "Amostragem com Probabilidade Proporcional a um Tamanho" ou (PPT).

Existem, entretanto, outras variações na formulação teórica deste processo, dependendo da maneira que a seleção das unidades amostrais é conduzida. Assim, se as unidades primárias são 
selecionadas com repetição, caracteriza um processo mais fácil para a derivação dos parâmetros e de seus estimadores, sem repetição. Nestas condições, a redução sucessiva das oportunidades de seleção para cada uma das unidades primárias, gera uma derivação de complexas fórmulas para os parâmetros e seus estimadores, estendendo-se também para o caso da subamostragem efetuada dentro das unidades primárias.

SUKHATME (1984) comenta que a concepção teórica para a seleção sem repetição, influi somente na estimativa da variância entre as unidades primárias e a diferença entre as duas concepções com e sem repetição, não é significante do ponto de vista prático. Nestas circunstâncias, a opção pela formulação teórica derivada para a seleção com repetição para as unidades primárias e sem repetição para a subamostragem é a mais apropriada no momento. Na prática, a seleção será efetuada sem repetição, para facilitar a condução do trabalho de campo. Uma ilustração esquemática do processo está apresentado na Figura 1.

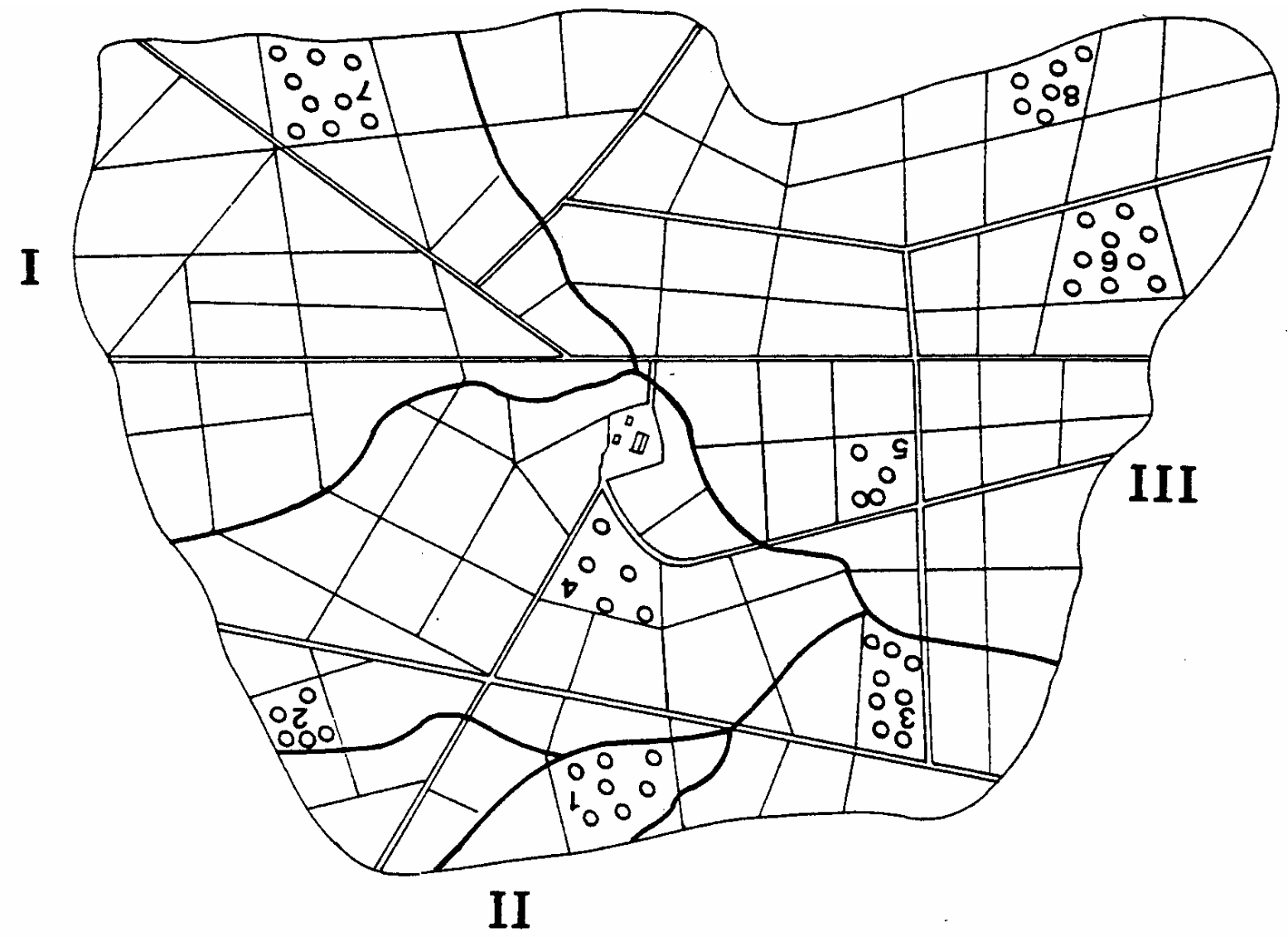

FIGURA 1: Ilustração esquemática da estrutura interna de um estrato com a alocação da subamostragem.

Como a seleção da amostragem será efetuada segundo o critério (PPT), então ficará caracterizada uma variável $Z_{i}$, que representará a proporção referente ao tamanho das unidades primárias em relação ao todo da área dentro de um estrato. Assim tem-se: 


$$
Z_{i}=\frac{M_{i}}{M}=\frac{A_{i}}{A}
$$

Onde: $\mathrm{M}_{\mathrm{i}}=$ Número de elementos dentro de cada unidade primária; $\mathrm{M}=$ Número total de elementos na área de um estrato; $\mathrm{A}_{\mathrm{i}}=$ Área de cada unidade primária; $\mathrm{A}=$ Área do estrato considerado.

Como mostrado anteriormente, em cada estrato, após a repartição ótima, resultará em $(m)$ unidades amostrais a serem alocadas nas unidades primárias segundo o critério (PPT). Esta repartição será efetuada organizando-se uma listagem das unidades primárias com seus respectivos tamanhos expressos por $\mathrm{M}_{\mathrm{i}}$ ou $\mathrm{A}_{\mathrm{i}}$.

Tomando-se a distribuição cumulativa de $\mathrm{M}_{\mathrm{i}}$, é possível obter os intervalos para cada unidade primária, cuja gama de variação permitirá, através de um sorteio inteiramente aleatório, receber uma alocação distributiva segundo o critério (PPT). Para efetivação desta seleção pode-se usar uma tabela de números aleatórios, onde as combinações de dígitos seriam utilizados dentro do intervalo total da gama de variação.

Para efeito de concretização da aplicação deste processo de amostragem, de tal forma a viabilizar uma análise de variância dentro do estrato, poder-se-á detectar as variabilidades internas e entre unidades primárias, se um mínimo de 4 subunidades for alocado por unidade primária, conforme propôs PÉLLICO NETTO (1979). Considerando-se esta restrição de que $\mathrm{m}_{\mathrm{i}} \geq 4$, pode-se proceder à repartição segundo o método $(\mathrm{PPT})$, tomando-se o total da amostragem $(\mathrm{m})$ e alocá-la sucessivamente em cada estrato, de tal forma que em cada unidade primária ter-se-á $\left(m Z_{\mathrm{i}}\right)$ unidades e os sucessivos sorteios continuarão até que se atinja o total $(m)$ a ser distribuído dentro do estrato.

A única restrição a esta seleção está no fato de que $(m)$ não será precisamente obtido no primeiro inventário, dado a variação total da população ainda não ser conhecida. Neste caso, uma estimativa inicial aproximada deverá ser feita e, posteriormente, pode ser corrigida à medida que a variação dentro dos estratos se torne bem conhecida, através da realização dos inventários sucessivos até à rotação.

\section{NOTAÇÃO E APRESENTAÇÃO DOS PARÂMETROS E ESTIMADORES DO PROCESSO DE AMOSTRAGEM}

As unidades amostrais primárias foram selecionadas com probabilidade proporcional a $\mathrm{Z}_{\mathrm{i}}$.

Seja a variável $\left(X_{\mathrm{ij}}\right)$ a medida do volume por elemento $j$, localizado na unidade primária $i$ (talhão). A notação básica para as unidades primárias fica assim definda como mostra a Tabela 1: 
TABELA 1: Notação básica para unidades primárias.

\begin{tabular}{lcc}
\hline VARIÁVEL & POPULAÇÃO & AMOSTRAGEM \\
\hline $\begin{array}{l}\mathrm{N}^{\mathrm{o}} \text { de elementos por unidades } \\
\text { primárias (Talhões) }\end{array}$ & $\mathrm{M}_{\mathrm{i}}$ & $\mathrm{m}_{\mathrm{i}}$ \\
Média por elemento & $\bar{X}_{i}=\frac{\sum_{j=1}^{M_{i}} X_{i j}}{M_{i}}$ & $\bar{x}_{i}=\frac{\sum_{j=1}^{m_{i}} X_{i j}}{m_{i}}$ \\
& $X_{i}=M_{i} \bar{X}_{i}$ & $\hat{X}_{i}=M_{i} \bar{x}_{i}$ \\
\hline
\end{tabular}

Para um estrato pode-se obter os resultados como está apresentado na Tabela 2.

TABELA 2: Notação básica para um estrato.

\begin{tabular}{|c|c|c|}
\hline VARIÁVEL & POPULAÇÃO & AMOSTRAGEM \\
\hline $\begin{array}{l}\text { Unidades primárias } \\
\text { no estrato (Talhões) }\end{array}$ & $\mathrm{N}$ & $\mathrm{n}$ \\
\hline $\mathrm{N}^{\circ}$ de elementos & $M=\sum_{i=1}^{N} M_{i}$ & $m=\sum_{i=1}^{n} m_{i}$ \\
\hline Média por elemento & $\overline{\bar{X}}=\frac{X}{M}$ & $x=\frac{x}{m}$ \\
\hline $\begin{array}{l}\text { Média por unidade primária } \\
\text { (talhão) }\end{array}$ & $\overline{\bar{X}}=\frac{1}{M} \sum_{i=1}^{N} \sum_{j=1}^{M} X_{i j}$ & $\bar{x}=\frac{1}{m} \sum_{i=1}^{n} \sum_{j=1}^{m} X_{i j}$ \\
\hline Total & $X=\sum_{i=1}^{N} X_{i}$ & $x=\sum_{i=1}^{n} X_{i}$ \\
\hline
\end{tabular}

Procedendo-se a realização da amostragem segundo o método de seleção (PPT), seus estimadores, de acordo com COCHRAN (1963) e SUKHATME et. al. (1984) podem ser obtidos como segue: ${ }_{x}(p p t)=\frac{1}{n M} \sum_{i=1}^{n} \frac{M_{i} \bar{x}_{i}}{Z_{i}}$ 
e sua variância é dada por:

$$
\underset{x(p p t)}{S_{x}^{2}}=\frac{1}{n M^{2}} \sum_{i=1}^{N} Z_{i}\left(\frac{X_{i}}{Z_{i}}-X\right)^{2}+\frac{1}{n M^{2}} \sum_{i=1}^{N} \frac{M_{i}^{2}}{Z_{i}}\left(\frac{1}{m_{i}}-\frac{1}{M_{i}}\right) S_{i}^{2}
$$

Onde:

$$
S_{i}^{2}=\frac{1}{M_{i}} \sum_{j=1}^{M_{i}}\left(X_{i j}-\bar{X}_{i}\right)^{2}
$$

Observe que se a amostragem for efetuada tomando-se $Z_{i}=\frac{M_{i}}{M}$, então a média definida em (2) reduz-se para:

$$
\bar{x}_{(p p t)}=\frac{1}{n M} \sum_{i=1}^{n} \frac{M M_{i} \bar{x}_{i}}{M_{i}}=\frac{1}{n} \sum_{i=1}^{n} \bar{x}_{i}
$$

e sua variância reduz-se para:

$$
\underset{x_{(p p t)}}{\underline{2}_{n}}=\frac{1}{n} \sum_{i=1}^{N} \frac{M_{i}}{M}\left(\bar{X}_{i}-\overline{\bar{X}}\right)^{2}+\frac{1}{n_{i=1}} \sum_{i=1}^{N} \frac{M_{i}}{M}\left(\frac{1}{m_{i}}-\frac{1}{M_{i}}\right) S_{i}^{2}
$$

Devido ao fato de que neste processo os estimadores dentro das unidades primárias são autoponderados, Cochran (1963) e Sukhatme (1984) demostraram que, para a amostragem, a variância da média é obtida como segue:

$$
\left.s_{\underset{x(p p t)}{\stackrel{2}{=}}=\frac{1}{n(n-1)} \sum_{i=1}^{n}\left(\bar{x}_{i}-{ }_{x}=(p p t)\right.}\right)^{2}
$$

\section{ILUSTRAÇÃO DO PROCESSO DE AMOSTRAGEM COM UNIDADES PRIMÁRIAS DE TAMANHOS DESIGUAIS A UMA POPULAÇÃO DE Pinus elliottii.}

Seja apresentado em seguida um exemplo ilustrativo do processo de seleção dentro de um estrato. Este exemplo, conquanto constitui-se de uma simulação, está ilustrado com dados reais de uma povoamento de Pinus elliottii. O estrato possui 32 unidades primárias (talhões), dos quais 8 foram selecionados e 210 elementos, dos quais 89 foram amostrados dentro dos talhões, ou seja $\mathrm{m}=89$. A distribuição da amostragem está apresentada na Tabela 3 , onde: $\propto$ - proporcional; Restrição $m_{i} \geq 4$.

Aplicando-se os estimadores aos estimadores da Tabela 3 tem-se: 
Média por elemento ou da subamostragem

$$
=\bar{x}_{(p p t)}=\frac{1}{n} \sum \bar{x}_{i}=\frac{176,533}{8}=22,066 \mathrm{~m}^{3} / 0,1 \mathrm{ha}
$$

\section{Variância da média da subamostragem}

$$
s_{x_{(p p t)}^{2}}^{=}=\frac{1}{n(n-1)} \sum_{i=1}^{n}\left(\bar{x}_{i}-{ }_{x}^{x}(p p t)\right)^{2}
$$

TABELA 3: Ilustração da Alocação da Subamostragem dentro de uma Estrato com Aplicação

\begin{tabular}{|c|c|c|c|c|c|c|}
\hline $\begin{array}{l}\text { UNIDADES } \\
\text { PRIMÁRIAS } \\
\text { (TALHÕES) }\end{array}$ & $\begin{array}{c}\text { ÁREAS } \\
\mathrm{A}_{\mathrm{I}} \\
\mathrm{m}^{2}\end{array}$ & $\begin{array}{c}\text { TAMANH } \\
\text { O } \\
\text { M }_{\mathrm{I}} \\
(0,1 \text { ha })\end{array}$ & $\begin{array}{c}\text { SELEÇÃO } \\
\propto \quad Z_{\mathrm{I}} \\
\mathrm{m}_{\mathrm{i}}\end{array}$ & $\begin{array}{c}\text { ORDEM } \\
\text { DA } \\
\text { SELEÇÃO }\end{array}$ & $\begin{array}{c}\text { MÉDIA } \\
\left(\mathrm{m}^{3} / 0,1 \mathrm{ha}\right)\end{array}$ & $\begin{array}{l}\text { VARIÂNCIA } \\
\left(\mathrm{m}^{3} / 0,1 \mathrm{ha}\right)^{2}\end{array}$ \\
\hline T-09 & 20.000 & 20 & 9 & 1 & 25,275 & 7,576 \\
\hline $\mathrm{T}-08$ & 20.000 & 20 & 9 & 2 & 21,475 & 4,442 \\
\hline $\mathrm{T}-05$ & 25.000 & 25 & 12 & 3 & 16,520 & 25,842 \\
\hline $\mathrm{T}-12$ & 30.000 & 30 & 14 & 4 & 26,100 & 5,676 \\
\hline $\mathrm{T}-10$ & 15.000 & 15 & 7 & 5 & 25,200 & 3,040 \\
\hline T-06 & 30.000 & 30 & 14 & 6 & 18,067 & 9,723 \\
\hline $\mathrm{T}-03$ & 35.000 & 35 & 17 & 7 & 14,129 & 35,199 \\
\hline $\mathrm{T}-18$ & 15.000 & 15 & 7 & 8 & 29,767 & 20,103 \\
\hline Total & 210.000 & $M=210$ & 89 & - & 176,533 & - \\
\hline
\end{tabular}
do Critério (PPT).

$$
\begin{aligned}
& s_{x(p x t)}^{\underline{2}}=\frac{1}{8(7)}\left[(25,275-20,562)^{2}+(21,475-20,562)^{2}+\ldots \ldots .+1\right] \\
& s_{x(p p t)}^{\stackrel{2}{=}}=\frac{1}{56} 205,794=3,675\left(m^{3} / 0,1 h a\right)^{2}
\end{aligned}
$$

\section{Erro padrão da estimativa}

$$
s_{x(p p t)}^{=}=\sqrt{3,675}=1,917 \mathrm{~m}^{3} / 0,1 \mathrm{ha}
$$




\section{Erro de amostragem}

$$
E_{a}= \pm s_{s_{(p p t)}}^{=}= \pm(2,306)(1,917)= \pm 4,421 \mathrm{~m}^{3} / 0,1 \mathrm{ha}
$$

\section{Erro de Amostragem Relativo}

$$
E_{r}=\frac{t s_{x(p p t)}^{=}}{x(p p t)} 100=\frac{4,421}{22,066} 100=20 \%
$$

Como observado, o erro exprimirá exatamente $10 \%$ do volume estimado para cada lado do intervalo de confiança.

\section{Intervalo de confiança para o valor paramétrico da média por subamostragem, para uma} confiabilidade de $95 \%$

$$
\begin{aligned}
& I C=\left[\overline{\bar{X}}=\bar{x}_{(p p t)} \pm E_{a}\right]=95 \% \\
& I C=[17,645 \leq \overline{\bar{X}} \leq 26,487]=95 \%
\end{aligned}
$$

\section{CONCLUSÕES}

O objetivo central deste trabalho foi atingido pela aplicação de uma metodologia de distribuição da amostragem dentro de um estrato florestal, considerando-se os talhões como parte integrante dos estimadores estatísticos e com alocação da subamostragem proporcional aos seus tamanhos respectivamente.

Pode-se ainda concluir que:

1. Especificando-se uma restrição de alocação mínima de 4 subunidades por talhão amostrado, visando-se permitir analisá-lo em suas variações internas pela aplicação de análise de variância, não será amostrado em cada ocasião a totalidade dos talhões e sua seleção para a amostragem será efetuada por sorteio aleatório, visando-se garantir a distribuição espacial sem tendência destes dentro do estrato. Tal procedimento, embora excluindo-se uma parte dos talhões na amostragem, pode ser equacionado em sucessivas ocasiões do inventário contínuo e será tratado em outro trabalho científico específico para este assunto;

2. Pela intensidade amostral utilizada na ilustração, aplicada a um povoamento de Pinus sp., localizada no Estado do Paraná, permite concluir que a metodologia usada é muito eficiente, dado que a distribuição das subunidades sendo efetuada proporcional à área dos talhões, resulta em uma autoponderação no cálculo do erro de amostragem e, assim, a torna recomendável para uso nos inventários contínuos de florestas plantadas; 
3. Para efeito de minimização de custos dos inventários contínuos, usando-se esta metodologia de alocação da subamsotragem, as unidades amostrais devem ser de pequeno tamanho para compensar o aumento de sua intensidade distributiva dentro dos talhões. Sugere-se, neste caso, a utilização de unidades, cujo critério probabilístico de seleção das árvores seja proporcional a um tamanho especificado, como é caso da unidade de Strand ou a de Prodan.

\section{REFERÊNCIAS BIBLIOGRÁFICAS}

COCHRAN, W. G. Sampling techniques. 2. ed., New York, John Wiley \& Sons, Inc., 1963. 413 p.

FAO. Manual of forest inventory with special reference to mixed tropical forests. Roma, FAO., 1973. $200 \mathrm{p}$.

PÉLLICO NETTO, S. Die Forstinventuren in Brasilien - Neue Entwicklungen und ihr Beitrag für eine geregelte Forstwirtschaft. Mitteilungen aus dem Arbeitskreis für Forstliche Biometrie. Freiburg, 1979. 232 p. (Tese de Doutorado).

SUKHATME et. al. Sampling theory of surveys, with applications. Ames, Iowa, Iowa State College Press. 1984. 526 p. 